\title{
ANÁLISE DAS ATIVIDADES DE EDUCAÇÃO EM SAÚDE REALIZADAS PELAS EQUIPES DE SAÚDE BUCAL
}

\author{
Analysis of health education activities performed by oral health teams \\ Análisis de las actividades de educación en salud realizadas por los equipos de salud bucal
}

\author{
Janice Dávila Rodrigues Mendes \\ Consórcio Público de Saúde da Microrregião de Sobral - CPSMS - Sobral (CE) - Brasil
}

\section{Cibelly Aliny Siqueira Lima Freitas}

Universidade Estadual Vale do Acaraú - UVA - Sobral (CE) - Brasil

Maria Socorro de Araújo Dias

Universidade Estadual Vale do Acaraú - UVA - Sobral (CE) - Brasil

\section{Mirna Marques Bezerra}

Universidade Federal do Ceará - UFC - Sobral (CE) - Brasil

José Jeová Mourão Netto

Hospital Regional Norte - HRN - Sobral (CE) - Brasil

Daniel Rodrigues Fernandes

Hospital Regional Norte - HRN - Sobral (CE) - Brasil

\section{RESUMO}

Objetivo: Analisar as atividades de Educação em Saúde realizadas pelas equipes de saúde bucal que estão inseridas na Estratégia Saúde da Família (ESF). Métodos: A pesquisa classificou-se em exploratória e descritiva, com abordagem qualitativa. O referencial teórico de Educação em Saúde utilizado foi a Educação Popular. O estudo foi realizado em Centros de Saúde da Família, Centro de Referência em Assistência Social (CRAS) e escolas no município de Sobral, Ceará, Brasil, entre os meses de novembro de 2013 e janeiro de 2014. Participaram do estudo 17 profissionais das equipes de saúde bucal. Os dados foram coletados por meio de entrevista contendo questões norteadoras e observação simples, sendo processados por meio da Análise do Discurso. Resultados: A concepção de Educação em Saúde referida majoritariamente pelos profissionais relaciona-se com o modelo tradicional. As atividades educativas realizadas não são sistematizadas para todo o município e estão condicionadas a programas ministeriais. A integração entre as equipes de saúde bucal e as equipes de saúde da família, no que diz respeito às atividades para escolares, é um processo ainda em construção, não estando consolidado na ESF. Conclusão: É necessário o desenvolvimento de políticas mais intensivas de formação de profissionais de saúde, que considerem a Educação Popular e o uso de metodologias ativas de ensino como método nas suas formações. Este estudo poderá contribuir para uma reflexão a respeito do tema na prática, podendo, assim, possibilitar a construção de um novo olhar sobre a Educação em Saúde.

Descritores: Educação em Saúde; Estratégia Saúde da Família; Saúde Bucal; Educação em Saúde Bucal.

\section{ABSTRACT}

Objective: To analyze the health education activities carried out by the oral health teams comprised in the Family Health Strategy (FHS). Methods: The research was classified as exploratory and descriptive, with a qualitative approach. Popular Education was adopted as the theoretical framework of Health Education. The study was performed at Family Health Centers, the Reference Center on Social Assistance (Centro de Referência em Assistência Social - CRAS) and schools in the municipality of Sobral, Ceará, Brazil, between November 2013 and January 2014. Seventeen professionals working in the oral health teams took part in the study. Data was collected through an interview with guiding questions and simple observation, and was processed through Discourse Analysis. Results: The conception of Health Education mostly referred to by the professionals is related to the traditional model. The educational activities carried out are not systematized for the whole municipality and are subject to ministerial programs. The integration between the oral health teams and the family health teams, with regard to activities for schoolchildren, is a process still under construction, not yet consolidated in the FHS. Conclusion: The development of more intensive policies for the training of health professionals, which consider Popular Education and the use of active teaching methodologies as method in their formation, is necessary. This study may contribute to a reflection regarding the theme in practice, thus enabling the construction of a new approach to Health Education.

Descriptors: Health Education; Family Health Strategy; Oral Health; Health Education Dental. 


\section{RESUMEN}

Objetivo: Analizar las actividades de educación en salud realizadas por los equipos de salud bucal inseridas en la Estrategia Salud de la Familia (ESF). Métodos: Se clasificó la investigación en exploratoria y descriptiva de abordaje cualitativo. El referencial teórico de educación en salud utilizado fue lo de Educación Popular. El estudio fue realizado en Centros de Salud de la Familia, Centro de Referencia en Asistencia Social (CRAS) y escuelas del municipio de Sobral, Ceará, Brasil entre los meses de noviembre de 2013 y enero de 2014. Participaron del estudio 17 profesionales del Equipo de Salud Bucal. Se recogieron los datos a través de entrevista con preguntas norteadoras y observación simples siendo procesados a través del Análisis del Discurso. Resultados: La concepción de Educación en Salud relatada por la mayoría de los profesionales se relaciona con el Modelo Tradicional de Educación en Salud. Las actividades educativas realizadas no son sistematizadas para todo el municipio y dependen de los programas ministeriales. Respecto las actividades para los escolares, la integración entre el Equipo de Salud Bucal y el Equipo de Salud de la Familia es un proceso todavía en construcción y no está consolidada en la ESF. Conclusión: Es necesario el desarrollo de políticas de formación de profesionales sanitarios más intensas que consideren la educación popular y el uso de las metodologías activas de enseñanza como método en sus formaciones. Ese estudio podrá contribuir para una reflexión sobre el tema en la práctica y asi posibilitar la construcción de una nueva mirada sobre la Educación en Salud.

Descriptores: Educación en Salud; Estrategia de Salud Familiar; Salud Bucal; Educación en Salud Dental.

\section{INTRODUÇÃO}

Os cuidados em saúde bucal fazem parte das ações preconizadas por instituições internacionais da meta de Saúde para Todos, criada desde 1977 e confirmada em 1978, na Declaração Internacional de Cuidados em Saúde de Alma-Ata. Essa meta foi atualizada sucessivamente, culminando recentemente nas Metas de Desenvolvimento do Milênio, estabelecidas em 2000. Contudo, a Organização Mundial de Saúde (OMS) publicou uma revisão global de saúde bucal enfatizando que, apesar de grandes melhorias na saúde bucal de populações em vários países, problemas persistentes ainda são graves, em particular nos grupos de menor poder aquisitivo, tanto em países em desenvolvimento quanto em países desenvolvidos ${ }^{(1)}$.

No que se refere à organização social da atenção em saúde bucal, especificamente no continente europeu, em países como a Alemanha, a atenção odontológica faz parte do catálogo de serviços do seguro social de doença, contudo, este é o setor assistencial em que incidem maiores taxas de copagamento. Os cirurgiões-dentistas credenciados atendem os segurados em seus consultórios particulares. Predominam os esquemas privados, com pagamentos por desembolso direto realizados pelos usuários ou esquemas de copagamento para alguns procedimentos cobertos pelas Caixas de Doença. Isenções de copagamento têm longa tradição no seguro social de doença alemão e são concedidas a grupos específicos da população, tais como pessoas de baixa renda ou desempregadas, pessoas com necessidades especiais, crianças e adolescentes até a idade de 18 anos e gestantes ${ }^{(2)}$.

$\mathrm{Na}$ Oceania, a Austrália possui um sistema de saúde cujo princípio fundamental é o acesso universal para a maioria da população, independentemente da capacidade de pagamento. Entretanto, serviços odontológicos em geral não são cobertos pela esfera pública, exceto para crianças e pessoas de baixa renda. A melhoria dramática em saúde bucal neste país, com a diminuição do índice de dentes permanentes cariados, perdidos e restaurados (CPO-D), tem sido atribuída à adição de flúor à água e a outros programas educativos e de promoção da saúde ${ }^{(3)}$.

Nas Américas, Cuba é um país que dispõe de um sistema de saúde inteiramente estatal, onde a saúde é um direito de todos e dever do Estado. Nos anos 80, com a introdução do modelo de atenção primária, baseado na medicina de família, houve a vinculação de dentistas de família a essa equipe de atenção. As ações são centradas na promoção da saúde e na prevenção de doenças bucais, para formar hábitos e estilos de vida saudáveis. Já nos Estados Unidos da América, o modelo de prestação de serviços odontológicos baseia-se essencialmente na prática individual privada, e o profissional recebe seus honorários diretamente do paciente. Uma ampla variedade de formas de pré e pós-pagamento proliferam no país, mantendo-se restrita a participação do setor público ${ }^{(2)}$.

Considerando que a saúde bucal é um componente vital da saúde, que contribui para o bem-estar e qualidade de vida, podendo ter fortes projeções biológicas, psicológicas e sociais ${ }^{(4)}$, houve uma reorganização das ações de saúde bucal no Brasil, de forma que as equipes de saúde bucal (ESB) integraram a Estratégia Saúde da Família (ESF) a partir dos anos 2000, com o objetivo de aprimorar a atenção à saúde e expandir o acesso às ações de saúde bucal, abonando a atenção integral aos indivíduos e às famílias. Foi regulamentada através da Portaria n. 1444, de dezembro de $2000^{(2)}$.

Em 2003, fundamentou-se uma Política Nacional de Saúde Bucal (PNSB), denominada Brasil Sorridente, com o propósito de estabelecer o direito do cidadão brasileiro à atenção em saúde bucal. Ela engloba ações individuais e coletivas para a promoção da saúde, a prevenção de agravos, o diagnóstico, o tratamento e a reabilitação. As ações de promoção e proteção da saúde visam minimizar fatores de risco que ameaçam a saúde das pessoas, possibilitando incapacidades e doenças. Envolvem 
as ações preventivo-curativas, fluoretação das águas, Educação em Saúde, higiene bucal supervisionada e aplicação tópica de flúor ${ }^{(5)}$

A Educação em Saúde engloba ações que enfocam o conhecimento sobre o processo saúde-doença, abrangendo fatores de risco e de proteção à saúde bucal, permitindo que a população adote mudança de hábitos e alcance a autonomia. A equipe de saúde deve estimular práticas de autocuidado por pacientes, famílias e comunidades ${ }^{(5)}$.

Entretanto, as ações de Educação em Saúde exigem uma compreensão ampliada de cuidado de saúde, sendo necessária a participação do usuário na mobilização, capacitação e desenvolvimento de aprendizagem de habilidades individuais e sociais para lidar com os processos de saúde-doença ${ }^{(6)}$.

Estudos mostram que, em relação à saúde bucal, a função do indivíduo é determinante, só estando em condições de enfrentar as várias afecções que podem incidir sobre sua saúde se tiver uma cultura sanitária que lhe permita praticar o autocuidado e a autorresponsabilidade ${ }^{(7)}$.

Portanto, as atividades educativas devem considerar a influência dos determinantes sociais, econômicos, políticos e culturais sobre as condições de vida e saúde da população. Apesar disso, estudos evidenciam que as ações de promoção da saúde ainda são utilizadas através de paradigmas tradicionais de intervenções educativo-preventivas no ambiente escolar, com foco na higiene bucal supervisionada, palestras e aplicações de fluoretos ${ }^{(8)}$.

Somando-se a esta problemática, a Educação em Saúde bucal vem sendo pouco descrita nos estudos de forma isolada, sendo as práticas mais avaliadas o flúor em dentifrícios e bochechos, assim como selantes oclusais. Há, ainda, um direcionamento dos estudos preventivos para grupos específicos, principalmente escolares. A regularidade e frequência do procedimento preventivo, assim como as ações voltadas aos diferentes problemas bucais e às outras faixas etárias, ainda são informações escassas ${ }^{(9)}$.

Diante do exposto, o trabalho de uma das autoras desse estudo como cirurgiã-dentista da ESF há quase 10 anos, a experiência como residente na residência multiprofissional em Saúde da Família (RMSF), instituída pela Escola de Formação em Saúde da Família Visconde de Saboia (EFSFVS), e a atuação como preceptora nesta, no município de Sobral, fez despertar o interesse sobre as práticas de promoção e prevenção em Odontologia, principalmente na área da Educação em Saúde, seja pela carência de estudos sobre estas práticas, seja pela falta de registros adequados destas ações, que impedem o seu acompanhamento longitudinal e não permitem avaliar o real impacto sobre a saúde bucal da população.

Na tentativa de superar o paradigma tradicionalmente realizado, pautado no modelo tecnicista e biomédico, historicamente determinado na formação da maioria dos profissionais da área da saúde, a estratégia de Educação Popular em Saúde ${ }^{(10)}$ pode contribuir com a promoção da saúde e a qualificação da Educação em Saúde, reforçando vínculos emancipatórios para que o indivíduo possa obter autonomia de como cuidar-se. Almejando fortalecer a mudança no modelo de atenção centrado na doença, é relevante a integração de serviços de Atenção Primária de Saúde às práticas populares de cuidado, pois estas dão uma visão de mundo e de saúde que se aproxima dos princípios que necessitam ser reforçados, como a integralidade, a humanização e o acolhimento ${ }^{(11)}$.

A Educação Popular em Saúde ${ }^{(10)}$ visa, através da obtenção do conhecimento, uma forma de superar as relações verticais conflitantes e modelos mecanicistas de apreciação da realidade social e implantação de novas propostas que recomendem a necessidade de mudança. Desse modo, se a promoção da saúde engloba os pilares de sustentação da ESF, conhecer como esta vem sendo trabalhada no âmbito das equipes de saúde bucal (ESB), pode facilitar a identificação de fragilidades e potencialidades, e auxiliar na melhoria das condições da atenção fornecida.

Desta forma, o presente trabalho teve como objetivo analisar as atividades de Educação em Saúde realizadas pelas equipes de saúde bucal inseridas na ESF.

\section{MÉTODOS}

Com base em seus objetivos, esta pesquisa classificou-se como exploratória e descritiva com abordagem qualitativa. O cenário do estudo aconteceu no município de Sobral, Ceará, Brasil, o qual integra uma rede assistencial hierarquizada e regionalizada do Sistema Único de Saúde (SUS), com capacidade instalada para realização de serviços em seus diferentes níveis de complexidade, que o faz ser município sede para a microrregional de Sobral e polo da macrorregião Norte do Ceará(12). Existem atualmente 57 ESF com 39 ESB, compostas por 39 cirurgiões-dentistas (CD) e 39 auxiliares de saúde bucal (ASB) ${ }^{(13)}$.

O município de Sobral foi um dos primeiros a implantar a ESB na ESF, em 1998, dando continuação ao Projeto de Reestruturação das Ações Odontológicas, apresentado em 1997 pela Secretaria de Desenvolvimento Social e Saúde. Foi um primeiro passo na obtenção de diagnóstico de situação, assinalando necessidades e proposições na área da saúde bucal ${ }^{(14)}$.

O Sistema Saúde Escola de Sobral, através da EFSFVS, contribui para a construção de novas tecnologias na atenção básica e na sistematização do fazer das diversas categorias profissionais, dando uma maior resolutividade no âmbito da atenção primária e consequentemente favorecendo o bem-estar da população. Não obstante, Sobral possui um histórico de doze anos investindo nessa modalidade de educação em serviço ${ }^{(15)}$. Muitos cirurgiões-dentistas do município cursaram a RMSF, portanto, existe uma notável evolução do serviço de saúde bucal, e muitas experiências exitosas já aconteceram durante todo este período, conforme descrito em experiências sistematizadas por egressos da $\operatorname{RMSF}^{(16,17)}$. 
O local do estudo consistiu em unidades básicas de saúde da família (UBS) que possuem ESB cadastradas e outros sítios onde os participantes realizam as atividades de Educação em Saúde, como o Centro de Referência em Assistência Social (CRAS) e as escolas. O período de coleta de dados perdurou do mês de novembro de 2013 a janeiro de 2014.

O universo da pesquisa foi composto por CD e ASB, que faziam parte das equipes de saúde da família no município de Sobral. Os critérios de inclusão dos CD para participação na pesquisa foram: estar há mais de um ano na ESF do município, restrito a somente um representante de cada UBS, atuar na sede do município, ou nos três distritos que se localizavam mais próximos geograficamente da sede, e com maior número de habitantes. Para os ASB, além dos mesmos critérios mencionados para os $\mathrm{CD}$, os critérios de inclusão consistiram em: ter concluído, ou estar realizando, o curso de ASB e trabalhar com o CD que estivesse participando da pesquisa. De acordo com esses critérios, teriam que participar da pesquisa $9 \mathrm{CD}$ e $9 \mathrm{ASB}$, mas, como um ASB estava de férias durante o período da coleta de dados, foram abordados $9 \mathrm{CD}$ e $8 \mathrm{ASB}$, compondo a amostra de 17 participantes.

Todos os participantes do estudo foram contatados previamente pelos pesquisadores, que expuseram os objetivos e a importância da pesquisa. Após o convite, para o aceite das partes, pactuou-se data e horário para a realização da coleta de dados com todos os representantes da amostra. Para a realização da coleta, utilizou-se entrevistas com as seguintes questões norteadoras: qual a sua percepção sobre Educação em Saúde? E como as atividades de educação em saúde são planejadas? Os instrumentos utilizados na técnica de observação simples foram um diário de campo e um check list, que auxiliaram na produção de um relatório minucioso das observações das atividades educativas.

O referencial teórico empregado foi o de Educação Popular, de Paulo Freire ${ }^{(10)}$, estratégia que deve ser utilizada para a formação de profissionais comprometidos com as questões sociais, mudando comportamentos e promovendo a reivindicação de direitos e comprometimentos, com atitudes acolhedoras e de construção da autonomia das pessoas e dos grupos sociais ${ }^{(11)}$.

$\mathrm{O}$ autor ${ }^{(10)}$ deixou como legado importantes reflexões sobre sujeitos marginalizados, deixando clara a relevância de se construir uma educação a partir do conhecimento do povo e com o povo, considerando a leitura da realidade na visão do oprimido, que se constitui nas relações históricas e sociais. Nesse sentido, o oprimido deve refletir e sair da condição de ser explorado a partir da estimulação da consciência de classe oprimida.

Dessa forma, os conteúdos de ensino, no método da Educação Popular, são denominados "temas geradores", sendo extraídos da problematização da prática de vida dos educandos. Os conteúdos tradicionais são recusados, porque cada pessoa, cada grupo envolvido na ação pedagógica dispõe em si próprio, ainda que de forma rudimentar, dos conteúdos necessários dos quais se parte. O importante não é a transmissão de conteúdos específicos, mas despertar uma nova forma da relação com a experiência vivida. A transmissão de conteúdos estruturados a partir de fora é considerada como "invasão cultural" ou "depósito de informação", porque não emerge do saber popular ${ }^{(18)}$.

As entrevistas foram realizadas nas UBS, mediante agendamento prévio, dentro dos consultórios odontológicos, como forma de garantir o sigilo e a confidencialidade dos profissionais, e de acordo com a disponibilidade de cada um. O tempo destinado às entrevistas deu-se conforme a necessidade dos participantes, sendo gravadas e, posteriormente, transcritas. Os profissionais entrevistados foram identificados em seus depoimentos por meio de letras iniciais de sua profissão e por números (CD1, CD2, ASB1, ASB2), como forma de garantir o seu anonimato.

A fase de observações simples ocorreu concomitantemente à realização das entrevistas com os profissionais de saúde bucal, totalizando cinco observações de atividades educativas desenvolvidas em diversos espaços: nas escolas municipais, que tiveram como público-alvo escolares na faixa etária de 4 a 7 anos; nas UBS, com público de gestantes e adultos; e no Centro de Referência em Assistência Social (CRAS), com pré-adolescentes e adolescentes de 8 a 12 anos. Realizaram-se duas observações das práticas de Educação em Saúde em escolas, duas em UBS e uma em CRAS.

No check list utilizado foi observado se os participantes empregavam abordagens dialógicas e participativas, se levavam a população a refletir criticamente sobre a realidade e se havia uma construção compartilhada do conhecimento, permitindo a interface com a cultura popular e a científica, segundo o referencial teórico empregado ${ }^{(10)}$.

A organização dos dados deu-se através do processo de categorização, sendo utilizado na análise o método de Análise do Discurso $^{(19)}$, do qual emergiu duas categorias: a primeira o referencial teórico de Educação em Saúde presente na percepção dos profissionais de saúde bucal, com suas subcategorias: modelo tradicional de Educação em Saúde e práticas educativas, que se aproximam da Educação Popular; e a segunda categoria, Educação em Saúde, com foco nas atividades da equipe de saúde bucal. Estas categorias serviram para orientar a análise, tendo sido definidas após a leitura flutuante das entrevistas e seguindo as orientações da autora de referência da Análise de Discurso.

A pesquisa foi desenvolvida de acordo com a Resolução n 466/12, do Conselho Nacional de Saúde ${ }^{(20)}$, respeitando seus aspectos éticos, e recebeu aprovação pelo Comitê de Ética em Pesquisa Local da Universidade Estadual Vale do Acaraú (UVA), sob o parecer número 470.588. Todos os participantes preencheram o Termo de Consentimento Livre e Esclarecido previamente à realização desta pesquisa. 


\section{RESULTADOS E DISCUSSÃO}

A seguir serão apresentadas as categorias temáticas e suas respectivas subcategorias, que emergiram do estudo.

\section{Referencial teórico de Educação em Saúde presente na percepção dos profissionais de saúde bucal Modelo tradicional de Educação em Saúde}

Essa subcategoria, conforme relatos dos profissionais, remete que a concepção de Educação em Saúde predominante nas entrevistas refere-se ao modelo tradicional de Educação em Saúde ou educação bancária ${ }^{(10)}$, na qual o profissional transmite o conteúdo, tido como verdade a ser absorvida; o usuário torna-se depósito de informações, sendo repassadas inúmeras recomendações sobre comportamentos "certos" e "errados", além de instruções de higiene oral, caracterizando uma transmissão verticalizada de conhecimento, refletindo no autoritarismo entre o educador e o educando ${ }^{(21)}$, como se pode notar através dos discursos a seguir:
"Para mim, Educação é tentar conscientizar a pessoa. Se a pessoa está consciente, é bem mais fácil o tratamento [...] Por mim, eu escovava os dentes de todo mundo, tipo, conscientizava e abria a cabecinha de todo mundo e colocava [...]”. (CD 5)
"Educação em Saúde, para mim, é tentar passar um pouco, né, para as pessoas sobre como elas podem se prevenir, em relação à saúde. Passar um pouco do básico [...], passar um pouco do que eu sei para aquelas pessoas, para que elas possam se prevenir [...]". (CD 4)
“É passar um pouco o que a gente sabe para alguém que não conhece [...]Então, eu estou promovendo saúde, não é? Acho assim que é uma maneira de educar o paciente para sua saúde bucal”. (ASB 4)

As atividades educativas realizadas por outros profissionais de saúde, como agentes comunitários de saúde (ACS) também são realizadas de acordo com esse modelo vertical de transmissão de informações ${ }^{(22)}$.

Outra pesquisa encontrou também a educação tradicional, na qual as enfermeiras demonstram alguns predicados do padrão hegemônico, regulado pela modelo sanitário prescritivo, com enfoque no controle das doenças e na intervenção e influência no comportamento das pessoas ${ }^{(23)}$.

Assim, desde a década de 1970, a Educação em Saúde vem sendo fortemente repensada, verificando-se um relativo distanciamento das ações impositivas, atributos do discurso higienista. Verifica-se uma ampliação do conhecimento do processo saúde-doença, que, saindo da concepção restrita do biologicismo, passa a ser visto como consequência da interrelação causal entre fatores sociais, econômicos e culturais. Neste momento, as práticas pedagógicas persuasivas, a transmissão verticalizada de conhecimento, permitindo o autoritarismo do educador para com o educando e a negação da subjetividade nos processos educativos, são passíveis de questionamentos. Surge, então, neste contexto, a preocupação com o desenvolvimento da autonomia, levando os sujeitos sociais a despertarem em busca de seus interesses ${ }^{(24)}$.

Assim, as intervenções educativas não devem visar apenas os indivíduos, mas verificar fatores que interfiram no comportamento em saúde e nas relações interpessoais, os fatores organizacionais e ambientais ${ }^{(25)}$. A consciência efetiva de uma mudança no comportamento em saúde é muito complexa e abrange diversas ações e ajustes ao longo do tempo ${ }^{(25)}$.

Durante as observações simples, verifica-se que o tema principal de todas as atividades refere-se à instrução de higiene oral, caracterizando simplesmente um repasse de informações para a população. Portanto, não há o estímulo para a construção da autonomia dos sujeitos nem o apoio para a co-responsabilização dos mesmos. Intervenções educativas fundamentadas em métodos tradicionais, como a simples transmissão de conhecimentos sem enfrentar os determinantes sociais dos comportamentos e do processo saúde-doença, são relevantes, mas poucas levam a mudanças de comportamento ${ }^{(25)}$.

Observou-se também que os métodos e instruções ensinados não são adequados à realidade de cada grupo, sendo iguais para todo o público-alvo participante. A população participante de abordagens educativas é capaz de intervir no processo de aprendizagem, devendo ser considerada influente e possuidora de atributos próprios. De tal modo, as metodologias de ensino devem adequar-se a cada realidade ${ }^{(26)}$.

Nesse sentido, a visão bancária de ensino dificulta um pensamento próprio. Educar não significa adestrar, mas aprender como um sujeito crítico, epistemologicamente curioso, que constrói o conhecimento ou participa de sua construção. É necessário não utilizar a transmissão bancária do conhecimento, mas ter ciência de que o educando tem experiências que devem ser consideradas $^{(10)}$.

O processo de formação profissional para o trabalho com a saúde da família, através do vários cursos de graduação ou formação continuada, ainda possuem ascendência no paradigma flexneriano de ensino, incompatível com o propósito da Educação em Saúde no contexto da $\operatorname{ESF}^{(27)}$.

As abordagens educativas com a utilização de grupos de pares treinados pela equipe podem, muitas vezes, incitar o desenvolvimento de comportamento em saúde oral, melhor do que quando demonstrado por profissionais ${ }^{(28)}$. 


\section{Práticas educativas que se aproximam da Educação Popular}

Nessa subcategoria, os participantes do estudo manifestaram um entendimento de Educação em Saúde de uma forma mais abrangente, aproximando-se da estratégia da Educação Popular: “A minha percepção sobre Educação em Saúde não é apenas assim fazer esse repasse de informação, e sim ter o cuidado
de trocar ideias, trocar informaçôes entre nós e a comunidade". (CD1)

"Eu acho que é o momento de você estar dividindo alguma coisa com alguém, que, no nosso trabalho, seria com a nossa comunidade, com os nossos escolares, com os nossos grupos. Então, assim, você está compartilhando o que você tem com outras pessoas, e também não serve só para você estar passando para a pessoa, porque a partir daquele momento é que você também ganha conhecimento [...]”. (CD 7)

Uma pesquisa ambientada no município de Sobral, Ceará, Brasil, evidenciou que práticas educativas dialógicas foram encontradas nas abordagens realizadas por enfermeiros, ainda que não fossem tão significativas. Comprovou igualmente que determinados profissionais, ao abordarem as orientações, partiam das necessidades e da realidade dos usuários, eximindo-se de conduta vertical ${ }^{(29)}$.

Em uma pesquisa realizada com profissionais de equipes de saúde da família do município de Santa Marina, Paraná, Brasil, objetivando analisar suas percepções sobre Educação em Saúde, apurou-se que alguns desses participantes adotam uma abordagem educativa pautada na reflexão, no diálogo e na problematização ${ }^{(30)}$.

A Educação em Saúde deve ser abordada visando a problematização habitual, considerando a vivência dos participantes, enquanto indivíduos, em grupos sociais e na contextualização das diferentes realidades. Como prática social, a Educação em Saúde passou a ser vista como uma estratégia que leva à reflexão e consciência crítica das pessoas sobre as origens de seus problemas de saúde, destacando o processo educativo abalizado no diálogo, de modo que se passe a trabalhar com as pessoas e não mais para as pessoas ${ }^{(31)}$.

Percebeu-se também, a partir dos discursos dos participantes, que a realização das atividades de Educação em Saúde é um processo desafiante para as equipes, como se pode verificar por meio da fala:

“Eu sou altamente a favor da Educação em Saúde. Eu gosto do que eu faço. Eu estou desestimulada, assumo, mas eu amo a minha profissão e amo também a saúde pública, embora seja um ramo muito difícil, que você tem que amar cinco vezes mais para conseguir trabalhar". (CD 5)

Durante as atividades de Educação em Saúde realizadas por esses profissionais, notou-se que havia uma combinação dos dois modelos educativos: tradicional e baseado na Educação Popular, na tentativa de superação do primeiro, já que ainda se apresentava fortemente inserido nas suas práticas profissionais.

Como uma maneira de suplantar a descontextualização das práticas pedagógicas, os acadêmicos devem ter espaço para aprender uma Educação em Saúde de forma dialógica e crítica, dentro do que determina a formação do perfil profissional, imprescindível para as práticas assistenciais vigorantes na ESF. Além disso, as estratégias pedagógicas pautadas na transdisciplinaridade e na atuação multiprofissional promovem a produção de novos conhecimentos, considerando o contexto social do complexo promoção-saúde-doença-cuidado ${ }^{(32)}$.

A Política Nacional de Educação Permanente em Saúde (PNEPS) orienta que a formação e o desenvolvimento de profissionais de saúde, respondendo às demandas do SUS, ocorram de modo descentralizado, ascendente e transdisciplinar, incluindo todos os locais e saberes, dando acesso à democratização dos espaços de trabalho ${ }^{(33)}$.

Neste sentido, torna-se importante enfatizar que essas reflexões não devem ficar restritas ao âmbito acadêmico. Os profissionais de saúde necessitam ser preparados para superar suas limitações e para o aperfeiçoamento e a atualização técnica a partir da educação permanente, que sugere um novo caminho para os profissionais de saúde produzirem conhecimento e pensarem a educação e o trabalho( ${ }^{(34)}$.

\section{Educação em Saúde: em foco as atividades da equipe de saúde bucal}

Nesta categoria, os profissionais da ESB, quando interrogados sobre como se dá o planejamento das atividades de Educação em Saúde, disseram que as atividades com foco nos escolares são planejadas com ajustes necessários; ocorrendo, ainda, integrado às atividades do Programa Saúde na Escola (PSE). Este programa proporciona o fortalecimento de ações com visão no desenvolvimento integral. Também, oportuniza a comunidade escolar a participação em projetos que articulem saúde e educação ${ }^{(34)}$.

"Os escolares, a gente planeja sempre no início do semestre para fazer as orientações, entrega de escovas e pastas, depois, de acordo com os programas, por exemplo, o PSE [...]. É semestral e depois de acordo com o PSE”. (CD 1)

Sobre a frequência dessas atividades para escolares, elas se dão sistematicamente, visando à meta do PSE, ou seja, devem ser feitos um número determinado de exames bucais e atividades educativas em cada escola: 
"Nas escolas, teve época que a gente ia até de três vezes por semana, que era para terminar o PSE, mas depois que encerrou, ai pronto, é uma vez no mês [...]Agora, em novembro, a gente não fez”. (ASB 7)

Contudo, apurou-se pelos discursos que as atividades são executadas de acordo com o planejamento de cada equipe. Não existe um planejamento para o município, de modo que cada equipe realiza da maneira que considera mais adequada, estando condicionadas às metas do PSE, ocorrendo com maior intensidade durante os meses de realização deste programa. Assim, não são práticas rotineiras, mas deveriam estar inseridas no processo de trabalho das ESB cotidianamente.

Ressalta-se, pelo discurso dos entrevistados, que são grandes os obstáculos que a equipe de saúde da família enfrenta para a execução das práticas cotidianas de ações educativas, pois são realizados fundamentalmente trabalhos em grupos com gestantes, hipertensos ou diabéticos, sendo as atividades de Educação em Saúde dirigidas de acordo com o programa ou a epidemia do momento ${ }^{(11)}$.

Quanto ao planejamento das atividades para escolares, a maioria referiu que é feito somente entre a ESB:

"As atividades para os escolares não, é só com a gente (o planejamento). A gente entra em contato com a gerência só para dizer os dias que a gente vai para as escolas”. (CD 4)

A dificuldade para realizar ações integradas com a ESF e ESB, ocorre, frequentemente, devido a problemas com agendas realizadas separadamente, inexperiência da ESB em lidar com a forma de atuação na ESF, ou, até mesmo, por falta de acordo entre ambas, como enfatizado no seguinte depoimento:

"A maior dificuldade para fazer estas atividades de educação é a falta de apoio da equipe (saúde da familia). Aqui é 'cada um no seu quadrado', ninguém ajuda na unidade. Tenho vontade de fazer mais atividades, planejar junto, mas não temos apoio, somos sempre deixados de lado”. (ASB 2)

Em pesquisa objetivando a análise da atenção à saúde bucal na ESF do Distrito Sanitário VI, em Recife, Pernambuco, Brasil, os profissionais entrevistados responderam que algumas atividades educativas entre a ESB e a ESF são pontuais, como nos grupos de hipertensos, diabéticos e de gestantes, mas, no geral, são totalmente desvinculadas ${ }^{(35)}$. Outros autores também enfatizaram que a integração da ESB com a de saúde da família para a realização de um trabalho em conjunto tem sido um dos maiores desafios para a saúde bucal na $\operatorname{ESF}^{(36)}$.

Acrescente-se, ainda, que as equipes têm pouco treinamento no contexto dos problemas de saúde bucal ${ }^{(37)}$, fato preocupante, pois a saúde bucal faz parte da atenção integral aos usuários. Além disso, os projetos pedagógicos devem reforçar cada vez mais a integração entre as equipes, visando a excelência do atendimento ${ }^{(38)}$.

A respeito da avaliação das ações educativas, mais relevante do que desenvolver ações coletivas de prevenção e Educação em Saúde bucal, é efetuá-las com avaliações periódicas, a fim de checar a efetividade e não instituir um "pacote preventivo", o qual tem sido implementado sem avaliação crítica pela maioria das $\mathrm{ESB}^{(36)}$.

A partir dos discursos, conclui-se que a avaliação não foi mencionada como uma das etapas do processo de desenvolvimento das atividades educativas; deste modo, essas atividades são planejadas e executadas de forma semelhante à metodologia do modelo tradicional de Educação em Saúde, em que as avaliações não são vistas como um instrumento de acompanhamento e monitoramento para a melhoria do desempenho das ações.

Diante do cenário até aqui descrito, percebe-se a necessidade de mais pesquisas sobre a Educação em Saúde bucal, visto que a literatura aponta a existência de lacunas em relação ao tema. Destaca-se, então, a necessidade de mais estudos sobre a temática que possam aprofundar e fundamentar a discussão e análise dos resultados, além de contribuírem para a consolidação da promoção da saúde bucal na ESF.

\section{CONSIDERAÇÕES FINAIS}

O referencial teórico de Educação em Saúde percebido pelos profissionais participantes desta pesquisa relaciona-se com o modelo tradicional de Educação em Saúde, visando principalmente mudanças individuais de comportamento, o qual guia metodologicamente suas práticas. Porém, no campo oposto, existe também uma percepção de Educação em Saúde mais abrangente, que se aproxima do modelo dialógico ou da Educação Popular, envolvendo o diálogo, a troca de experiências e o "aprender/estar com o outro". Observou-se que a aplicação dos princípios da Educação Popular na Saúde pode constituir um grande desafio para as ESB, visto que os profissionais podem não possuir conhecimento sobre esse modelo e não terem vivenciado momentos educativos baseados nessa metodologia.

Sobre o processo de planejamento e desenvolvimento, as atividades que têm como público-alvo os escolares são planejadas somente entre a ESB. Não há uma sistematização para todo o município e estão condicionadas e orientadas por um programa do Ministério da Saúde, em parceria com o Ministério da Educação, o PSE. Nota-se que a integração entre ESB e equipe de saúde da família, no que diz respeito às atividades para escolares, é um processo ainda em construção, não estando consolidado na ESF. 
O processo histórico de inserção da saúde bucal na ESF, a formação acadêmica dos profissionais, os programas ministeriais, com seus protocolos e metas, os aspectos estruturais e as concepções acerca da Educação em Saúde confluem para a organização de um processo de trabalho que privilegia o modelo tradicional de educação, o que faz com que este permaneça hegemônico nas práticas atuais.

Para a construção de um novo olhar sobre a Educação em Saúde, é necessário o desenvolvimento de políticas mais intensivas de formação de profissionais de saúde, desde a graduação, assim como em nível técnico, que considerem a Educação Popular e o uso de outras metodologias ativas de ensino como método nas suas formações. Para que as ESB possam usar a Educação Popular para estimular o empoderamento da população, sua autonomia para decidir sobre sua vida e sua saúde, é importante que ela seja utilizada como norteadora das educações permanentes dos trabalhadores.

\section{REFERÊNCIAS}

1. Petersen PE, Bourgeois D, Ogawa H, Estupinan-Day S, Ndiaye C. The global burden of oral diseases and risks to oral health. Bull World Health Organ. 2005;83(9):661-9.

2. Giovanella L, Escorel S, Lobato LVC, Noronha JC, Carvalho AI. Políticas e Sistema de Saúde no Brasil. $2^{\mathrm{a}}$ ed. Rio de Janeiro: Fiocruz; 2012.

3. Malmö University. Oral Health Country / Area Profile Project [Internet]. Malmö; 2011 [acesso em 2016 Maio 22]. Disponível em: http://www.mah.se/CAPP/

4. Relwani AH, Kiran S, Bhatt R, Patel M. Impact of dental health education on "Specific Learning Needs" Children. Int J Clin Pediatr Dent [Internet]. 2016 [acesso em 2016 Maio 27];9(1):31-4. Disponível em: https://www.ncbi.nlm.nih.gov/ pmc/articles/PMC4890059/

5. Ministério da Saúde (BR), Secretaria de Atenção à Saúde, Departamento de Atenção Básica. Coordenação Nacional de Saúde Bucal. Diretrizes da Política Nacional de Saúde Bucal. Brasília: Ministério da Saúde; 2004.

6. Machado MFAS, Vieira NFC. Educação em Saúde: O olhar da Equipe de Saúde da Família e a participação do usuário. Rev Latinoam Enferm. 2009;17(2):174-9.

7. Angulo EL, Cabrera LG, Fernández CG, Gross SC, Lugo FS. La cultura en salud bucal como problema actual de la sociedade. MEDISAN. 2013;17(4):677-85.

8. Matos CV, Gondinho BVC, Ferreira DLA. A Educação em Saúde Bucal e suas representações na Atenção Primária á Saúde. Rev Elet Gestão \& Saúde [Internet]. 2015 [acesso em 2016 Maio 27];6 (1):845-55. Disponível em: http://gestaoesaude. unb.br/index.php/gestaoesaude/article/view/1246

9. Almeida GCM, Ferreira MAF. Saúde Bucal no contexto do Programa Saúde da Família: práticas de prevenção orientadas ao individual e ao coletivo. Cad Saúde Publica. 2008;24(9):2131-40.

10. Freire P. Pedagogia do oprimido. Rio de Janeiro: Paz e Terra; 1987.

11. Ministério da Saúde (BR), Secretaria de Gestão Estratégica e Participativa. Caderno de Educação Popular em Saúde. Brasília: Ministério da Saúde; 2014.

12. Governo do Estado do Ceará, Secretaria da Saúde. Coordenadorias Regionais de Saúde [Internet]. Fortaleza; 2010 [acesso em 2016 Jun 02]. Disponível em: http://www.saude.ce.gov.br/index.php/regionalizacao

13. Ministério da Saúde (BR), Secretaria de Atenção à Saúde, DATASUS- Departamento de Informática do SUS. CNES NetCadastro Nacional de Estabelecimentos de Saúde. 2013 [acesso em 2013 Ago 03]. Disponível em: http://cnes2.datasus.gov. br/Lista_Es_Nome.asp?VTipo $=0$

14. Mendes FIR, Viana RS. A Percepção dos gerentes das unidades básicas de saúde sobre a inserção da equipe de saúde bucal na Estratégia de Saúde da Família em Sobral-CE. Sanare (Sobral). 2005;6(1):19-28.

15. Melo CNM, Chagas MIO, Feijão JRP, Dias MSA. Programa de Residência Multiprofissional em Saúde da Família de Sobral: uma avaliação de egressos a partir da inserção no mercado de trabalho. Sanare (Sobral). 2012;11(1):18-25.

16. Paresque MAC, Aragão DS, Fernandes MBL, Santos MLMF, Mendes JDR, Pereira PM. A atenção em saúde bucal e a operacionalização das suas práticas na Estratégia em Saúde da Família a partir da Residência Multiprofissional no município de Sobral- CE. Sanare (Sobral). 2009;8(2):73-82.

17. Paresque MAC, Taboza Z, Dias C, Francelino R. Ressignificando as práticas na atenção em saúde bucal aos escolares. Sanare (Sobral). 2011;10(1):49-53. 
18. Luckesi CC. Filosofia da Educação. $3^{\mathrm{a}}$ ed. São Paulo: Cortez; 2011.

19. Minayo MCS. O desafio do conhecimento: pesquisa qualitativa em saúde. $13^{\text {a }}$ ed. São Paulo: Hucitec; 2013.

20. Ministério da Saúde(BR), Conselho Nacional de Saúde, Comissão Nacional de Ética em Pesquisa. Normas regulamentadoras de pesquisa envolvendo seres humanos. Resolução nº 196/96- versão 2012. Brasília: Ministério da Saúde; 2012.

21. Cervera DPP, Parreira BDM, Goulart BF. Educação em saúde: percepção dos enfermeiros da atenção básica em Uberaba (MG). Ciênc Saúde Coletiva. 2011;16(Supl 1):1547-54.

22. Mialhe FL, Lefèvre F, Lefèvre AMC. O agente comunitário de saúde e suas práticas educativas em saúde bucal: uma avaliação qualiquantitativa. Ciênc Saúde Coletiva. 2011;16(11):4425-32.

23. Figueira MCS, Leite TMC, Silva EM. Educação em saúde no trabalho de enfermeiras em Santarém do Pará, Brasil. Rev Bras Enferm. 2012;65(3):414-9.

24. Vernon LT, Howard AR. Advancing Health Promotion in Dentistry: Articulating an Integrative Approach to Coaching Oral Health Behavior Change in the Dental Setting. Curr Oral Health Rep [Internet]. 2015 [acesso em 2016 Jun 02];2(3):111-2. Disponível em: https://www.ncbi.nlm.nih.gov/pmc/articles/PMC4596064/

25. Lemkuhl I, Souza MVC, Cascaes AM, Bastos JL. A efetividade das intervenções educativas em saúde bucal: revisão de literatura. Cad Saúde Colet (Rio J). 2015;23(3):336-46.

26. Inocente-Díaz ME, Pachas-Barrionuevo F. Educación para la Salud em Odontología. Rev Estomatol Hered. 2012;22(4):23241.

27. Mendes EV. O cuidado das condições crônicas na atenção primária à saúde: o imperativo da consolidação da estratégia da saúde da família. Brasília: Organização Pan-Americana da Saúde; 2012.

28. Vangipuram S, Jha A, Raju R, Bashyam M. Effectiveness of peer group and conventional method (dentist) of oral health education programme among 12-15 year old school children - a randomized controlled trial. J Clin Diagn Res [Internet]. 2016 [acesso em 2016 Jun 02];10 (5):152-9. Disponível em: https://www.ncbi.nlm.nih.gov/pmc/articles/PMC4948521/

29. Silva CP, Dias MAS, Rodrigues AB. Práxis educativa em saúde dos enfermeiros da Estratégia Saúde da Família. Ciênc Saúde Coletiva. 2009;14(Supl 1):1453-62.

30. Pinafo E, Nunes EFPA, González AD, Garanhani ML. Relações entre concepções e práticas de educação em saúde na visão de uma equipe de saúde da família. Trab Educ Saúde. 2011;9(2):201-21.

31. Alves GG, Aerts D. As práticas educativas em saúde e a Estratégia Saúde da Família. Ciênc Saúde Coletiva. 2011;16(1):31925.

32. Mialhe FL, Silva CMC. A educação em saúde e suas representações entre alunos de um curso de odontologia. Ciênc Saúde Coletiva. 2011;16 (Supl 1):1555-61.

33. Ministério da Saúde (BR), Ministério da Educação. Passo a Passo- PSE- Programa Saúde na Escola: tecendo caminhos da intersetorialidade. Brasília: Ministério da Saúde; 2011.

34. Silva CT, Terra MG, Kruse MHL, Camponogara S, Xavier MS. Residência Multiprofissional como espaço intercessor para a Educação Permanente em Saúde. Texto \& Contexto Enferm. 2016;25(1):1-9.

35. Pimentel FC, Martelli PJL, Araújo JLAC Júnior, Acioli RML, Macedo CLSV. Análise da atenção à saúde bucal na Estratégia de Saúde da Família do Distrito Sanitário VI, Recife (PE). Ciênc Saúde Coletiva. 2010;15(4):2189-96.

36. Souza TMS, Roncalli AG. Saúde bucal no Programa Saúde da Família: uma avaliação do modelo assistencial. Cad Saúde Publica. 2007;23(11):2727-39.

37. Cohen LA. Expanding the physician's role in addressing the oral health of adults. Am J Public Health. 2013;103(3):408-12.

38. Park SE, Donoff RB, Saldana F. The impact of integrating oral health education into a medical curriculum. Med Princ Pract [Internet]. 2017 [acesso em 2016 Ago 03];26:61-5. Disponível em: https:/www.karger.com/Article/ FullText/452275\#

\section{Endereço para correspondência}

Janice Dávila Rodrigues Mendes

Consórcio Público de Saúde da Microrregião de Sobral - CPSMS

Rua Padre Antônio Ibiapina, 170

Bairro: Centro

CEP: 62010-750 - Sobral - CE - Brasil

E-mail: janicedavila34@gmail.com 